\title{
AVALIAÇÃO DA NUTRIÇÃO ENTERAL EM UNIDADE DE TERAPIA INTENSIVA
}

\author{
EVALUATION OF ENTERAL NUTRITION \\ IN AN INTENSIVE CARE UNIT
}

\section{EVALUACIÓN DE LA NUTRICIÓN ENTERAL EN UNA UNIDAD DE CUIDADOS INTENSIVOS}

\author{
Sterline Therrier ${ }^{1}$ \\ Clara Marize Carlos ${ }^{2}$ \\ Raquel Fernandes Costa ${ }^{3}$ \\ Giovana Paula Rezende Simino ${ }^{4}$ \\ Jaqueline Almeida Guimarães Barbosa ${ }^{5}$
}

Como citar este Artigo: Therrier S, Carlos CM, Costa RF, Simino GPR, Barbosa JAG. Avaliação da nutrição enteral em unidade de terapia intensiva. Rev baiana enferm. 2021;35:e38558.

Objetivo: avaliar a infusão da nutrição enteral em pacientes adultos internados em unidade de terapia intensiva. Método: estudo descritivo, quantitativo e longitudinal, desenvolvido ao longo de quatro meses em hospital público de alta complexidade, com pacientes em uso da terapia nutricional enteral. Foram realizadas análises estatísticas descritivas com média, desvio-padrão e percentual. Resultados: a administração da nutrição enteral ocorreu de forma precoce após a admissão na unidade de terapia intensiva na maioria dos casos (64\%). Em $71 \%$ dos pacientes, o volume de infusão da nutrição enteral foi entre 80 e 100\% do prescrito. Os fatores que mais limitaram a infusão foram complicações no uso da sonda de alimentação (14\%), instabilidade hemodinâmica e clínica (12\%), e estase gástrica (12\%). Conclusão: a infusão da nutrição enteral mostrou-se satisfatória, e dentre os fatores que limitaram a infusão muitos são passíveis de prevenção com o aprimoramento dos cuidados de enfermagem, requerendo a capacitação da equipe.

Descritores: Nutrição Enteral. Unidades de Terapia Intensiva. Cuidados de Enfermagem.

Objective: to evaluate the infusion of enteral nutrition in adult patients admitted to an intensive care unit. Method: a descriptive, quantitative and longitudinal study, carried out over four months in a bigh-complexity public hospital, with patients using enteral nutritional therapy. Descriptive statistical analyses were performed with mean, standard deviation and percentage. Results: enteral nutrition was administered early after admission to the intensive care unit in most of the cases (64\%). In $71 \%$ of the patients, the infusion volume of enteral nutrition was between $80 \%$ and $100 \%$ of that prescribed. The factors that most limited the infusion were complications in the use of the feeding tube (14\%), hemodynamic and clinical instability (12\%), and gastric stasis (12\%). Conclusion: the infusion of

\footnotetext{
Enfermeira. Escola de Enfermagem da Universidade Federal de Alfenas. Alfenas, Minas Gerais, Brasil. sterlinetherrier@yahoo.fr. http://orcid.org/0000-0002$7713-3288$.

2 Enfermeira. Mestre em Enfermagem. Especialista em Terapia Nutricional. Enfermeira do Hospital das Clínicas da Universidade Federal de Minas Gerais. Belo Horizonte, Minas Gerais, Brasil. http://orcid.org/0000-0002-9908-507X.

3 Enfermeira. Especialista em Terapia Nutricional. Enfermeira do Hospital das Clínicas da Universidade Federal de Minas Gerais. Belo Horizonte, Minas Gerais, Brasil. http://orcid.org/0000-0002-6276-47| 4

4 Enfermeira. Doutora em Enfermagem. Professora Adjunta do Departamento de Enfermagem Básica da Escola de Enfermagem. Universidade Federal de Minas Gerais. Belo Horizonte, Minas Gerais, Brasil. http://orcid.org/0000-0002-9814-3004.

Enfermeira. Doutora em Enfermagem. Professora Adjunta do Departamento de Enfermagem Básica da Escola de Enfermagem. Universidade Federal de Minas Gerais. Belo Horizonte, Minas Gerais, Brasil. http://orcid.org/0000-0002-9|75-0055.
} 
enteral nutrition proved to be satisfactory and, among the factors that limited the infusion, many are preventable with the improvement of Nursing care, requiring the training of the team.

Descriptors: Enteral Nutrition. Intensive Care Units. Nursing Care.

Objetivo: evaluar la infusión da nutrición enteral en pacientes adultos internados en una unidad de cuidados intensiva. Método: estudio descriptivo, cuantitativo y longitudinal, desarrollado en el transcurso de cuatro meses en un hospital público de alta complejidad, con pacientes sometidos a terapia nutricional enteral. Se realizaron análisis estadísticos descriptivos con media, desvio estándar y porcentaje. Resultados: la administración de la nutrición enteral tuvo lugar en forma temprana después del ingreso a la unidad de cuidados intensivos en la mayoria de los casos (64\%). En el $71 \%$ de los pacientes, el volumen de infusión de la nutrición enteral fue entre el $80 \%$ y el $100 \%$ de lo prescripto. Los factores que más limitaron la infusión fueron complicaciones en el uso de la sonda de alimentación (14\%), inestabilidad hemodinámica y clinica (12\%), y estasis gástrica (12\%). Conclusión: la infusión de la nutrición enteral evidenció ser satisfactoria y, entre los factores que limitaron la infusión, muchos son pasibles de prevenirse con una mejora de la atención de Enfermería, lo que requiere capacitación del equipo.

Descriptores: Nutrición Enteral. Unidades de Cuidados Intensivos. Atención de Enfermería.

\section{Introdução}

A desnutrição em pacientes hospitalizados é uma realidade mundial, demonstrada em vários estudos. Em uma revisão sistemática ${ }^{(1)}$ na qual foram incluídas 66 pesquisas, realizadas em 12 países da América Latina, englobando 29.474 pacientes, encontrou-se que a prevalência da desnutrição variou entre 40 e 60\% no momento da admissão. Essa prevalência aumenta ainda mais ao longo da internação, decorrente de fatores diversos, como aqueles relacionados à própria doença em fase aguda e os relacionados ao tratamento. Essa situação é motivo de grande preocupação devido a sua correlação com o aumento da morbidade, repercutindo em cicatrização mais lenta de feridas, taxa de infecção aumentada, aumento do tempo de internação e reinternação, bem como aumento de custos, além da mortalidade ${ }^{(1)}$.

Esse contexto é ainda mais complexo quando se trata de pacientes internados em Unidade de Terapia Intensiva (UTI), quando permanecem, na maioria das vezes, impossibilitados de se alimentarem por via oral. Soma-se a isso o estresse propiciado pelo estado crítico em que se encontram, que desencadeia alterações hormonais que, por sua vez, provocam hipercatabolismo, com perdas proteicas e calóricas significativas, favorecendo a ocorrência ou a piora da desnutrição ${ }^{(2)}$.
Para enfrentar a problemática da desnutrição hospitalar e/ou atenuar seu impacto nos pacientes previamente impossibilitados ou incapazes de manter a ingestão por via oral, dispõem-se do recurso da terapia nutricional, que pode ser oferecida por meio da via oral, enteral ou parenteral. A Terapia Nutricional Enteral (TNE) vem sendo amplamente utilizada nos hospitais brasileiros, e requer que o paciente tenha o trato gastrointestinal preservado e funcionante para que seja administrada.

A Nutrição Enteral (NE) pode ser infundida por meio do cateter de alimentação, mais conhecido por sonda nasoenteral (SNE), termo este adotado a fim de se evitar confusões com outros cateteres, impedindo, assim, possíveis erros. A NE pode ser infundida ainda por meio de ostomias de alimentação (gastro ou jejunostomias) em pacientes que necessitam dessa terapêutica por longo período, superior a quatro semanas ${ }^{(3)}$.

A infusão de alimentos através do trato gastrointestinal, além de mais fisiológica, é fundamental para manter-se a integridade metabólica e imunológica do organismo. Além disso, quanto mais precocemente for iniciada nos pacientes em tratamento intensivo, melhor é o desfecho, com redução considerável das taxas de complicações e do risco de mortalidade. A terapia nutricional enteral precoce consiste em iniciar a prescrição 
e a infusão da nutrição enteral em até 48 horas e, preferencialmente, nas primeiras 24 horas após a internação na unidade de terapia intensiva ${ }^{(4-5)}$.

Entretanto, estudos mostram que um número considerável de pacientes internados em unidade de terapia intensiva recebe um volume de nutrição enteral inferior ao prescrito ou com início tardio, comprometendo a recuperação dos pacientes. Essa problemática é justificada por fatores, como a instabilidade hemodinâmica, uso de drogas vasoativas e decorrentes de realização de procedimentos médicos, de enfermagem e de fisioterapia durante o período de internação e também interrupções na infusão da dieta decorrentes de intercorrências gastrintestinais, problemas com a sonda de alimentação, bem como atrasos em ligar a nutrição enteral ${ }^{(6-7)}$.

A infusão da NE é responsabilidade da enfermagem para suprir a necessidade humana básica de nutrição. Cabe a este profissional atentar-se para todos os aspectos que possam ocasionar atrasos no procedimento, evitando-os ao máximo, diante da importância da nutrição para o restabelecimento do estado de saúde e prevenção de complicações, principalmente em pacientes críticos. Assim, faz-se necessário monitorar a infusão da nutrição enteral nas diferentes instituições, identificando fragilidades no processo que possam comprometer o fornecimento do aporte planejado para os pacientes, o que pode variar conforme o perfil da instituição.

Dessa forma, o objetivo deste trabalho foi avaliar a infusão da nutrição enteral em pacientes adultos internados em unidade de terapia intensiva. Espera-se contribuir com informações que favoreçam o aprimoramento da prática assistencial visando a recuperação dos pacientes.

\section{Método}

Estudo descritivo, longitudinal, com abordagem quantitativa. Foi realizado o monitoramento da infusão da NE em todos os pacientes com idade superior ou igual a 18 anos, que se encontravam em uso da terapêutica, internados na unidade de terapia intensiva, sendo estes os critérios de inclusão. Foram excluídos os pacientes que permaneceram menos de 72 horas em uso de nutrição enteral e aqueles cujas informações, por motivos diversos, não puderam ser coletadas na íntegra.

A unidade de terapia intensiva onde se deu a coleta dos dados está inserida em um hospital geral, de ensino público e de grande porte, e é referência no atendimento de média e alta complexidade no estado de Minas Gerais. A instituição conta com aproximadamente 540 leitos. A unidade de terapia intensiva tem 90 leitos; os cuidados aos pacientes são prestados essencialmente por enfermeiros graduados. O hospital tem Equipe multiprofissional de terapia nutricional bastante atuante, que estabelece os protocolos assistenciais e dá suporte aos enfermeiros da linha de frente em todos os aspectos relacionados à terapia nutricional, bem como monitora a sua efetividade.

A coleta de dados foi realizada diariamente por uma acadêmica de enfermagem devidamente capacitada, ao longo de quatro meses (agosto a novembro de 2017). A coleta deu-se no primeiro dia de início de uso da terapia nutricional enteral até a sua suspensão definitiva, seja por motivo de restituição da via oral, alta da unidade de terapia intensiva ou óbito. A fim de caracterizar a população do estudo foram colhidas informações de cunho sociodemográfico (sexo e idade), dados clínicos, como diagnóstico de internação e desfecho do paciente acompanhado, além de dados relacionados ao início do uso da nutrição enteral, se houve interrupção da infusão e suas causas, e o balanço final da infusão ocorrida. Todos os dados foram coletados dos prontuários e registrados em formulário próprio desenvolvido especialmente para este estudo.

É protocolo institucional que todo paciente em unidade de terapia intensiva e em terapia nutricional tenha seu balanço hídrico realizado diariamente, parcialmente fechado a cada seis horas. Assim, a bomba de infusão contínua usada para administração da nutrição enteral é zerada e a programação reiniciada, possibilitando quantificar o volume de NE administrado. Além disso, o cálculo do volume prescrito é feito considerando a programação de infusão em 20 horas ao 
dia, já antevendo quatro horas de interrupções para a prestação de cuidados e a realização de procedimentos diversos, como atendimento da equipe multiprofissional.

Os dados foram digitados e analisados no Excel versão 2013. Foram realizadas análises estatísticas descritivas de frequência percentual e relativa, e cálculos de média e desvio-padrão para as variáveis contínuas.

O estudo teve a anuência da instituição envolvida e também foi aprovado pelo Comitê de Ética em Pesquisa (CEP) da Universidade Federal de Minas Gerais (UFMG) obtendo Parecer n. 2.232.124, CAAE: 72683417.3.0000.5149, e respeitando-se as orientações da Resolução n. 466/12, do Conselho Nacional de Saúde.

\section{Resultados}

Inicialmente foram incluídos 107 pacientes em uso de terapia nutricional enteral para acompanhamento; destes, 28 foram excluídos por deixarem de atender a algum critério pré-definido. Ao final, o estudo contou com a análise da infusão da nutrição enteral de 79 pacientes, dentre os quais 50,6\% eram do sexo feminino. A idade máxima dos pacientes acompanhados foi de 88 anos e a mínima de 18, a média de idade em anos foi de 56,8 $\pm 18,88$. Dentre as causas de internação na unidade de terapia intensiva prevaleceram as doenças neurológicas (34\%), seguida das cardíacas (19\%), respiratórias (12\%), sepse (10\%), outros (9\%), pós-operatório (6\%), neoplasias e transplantes (5\% ambos).

Quanto ao início da nutrição enteral em relação à admissão do paciente na unidade de terapia intensiva, este se deu em média com 2,8 2,4 dias de internação, o tempo mínimo para se dar o início foi de 1 dia e o máximo de 11 dias. A maioria (64.6\%) começou a receber a NE com 1 e 2 dias após a internação na unidade de terapia intensiva (Gráfico 1).

Gráfico 1 - Início da Nutrição Enteral em relação à admissão do paciente na Unidade de Terapia Intensiva. Belo Horizonte, Minas Gerais, Brasil - 2017. (N=79)

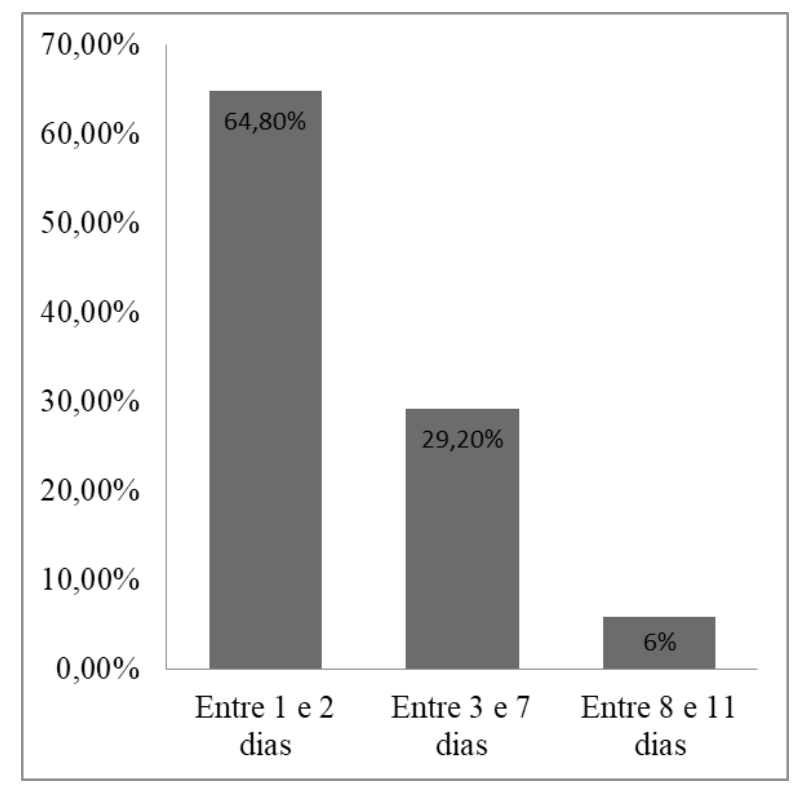

Fonte: Elaboração própria.

De acordo com o Gráfico 1, identificou-se uma média de 12,3 $\pm 11,8$ dias de uso da terapia nutricional enteral. Quanto ao balanço da infusão da nutrição enteral, $71 \%$ dos pacientes acompanhados atingiram o percentual entre 80 e 100\% do volume prescrito, conforme ilustrado no Gráfico 2. 
Gráfico 2 - Balanço da Infusão da Nutrição Enteral em Unidades de Terapia Intensiva. Belo Horizonte, Minas Gerais, Brasil - 2017. (N=79)

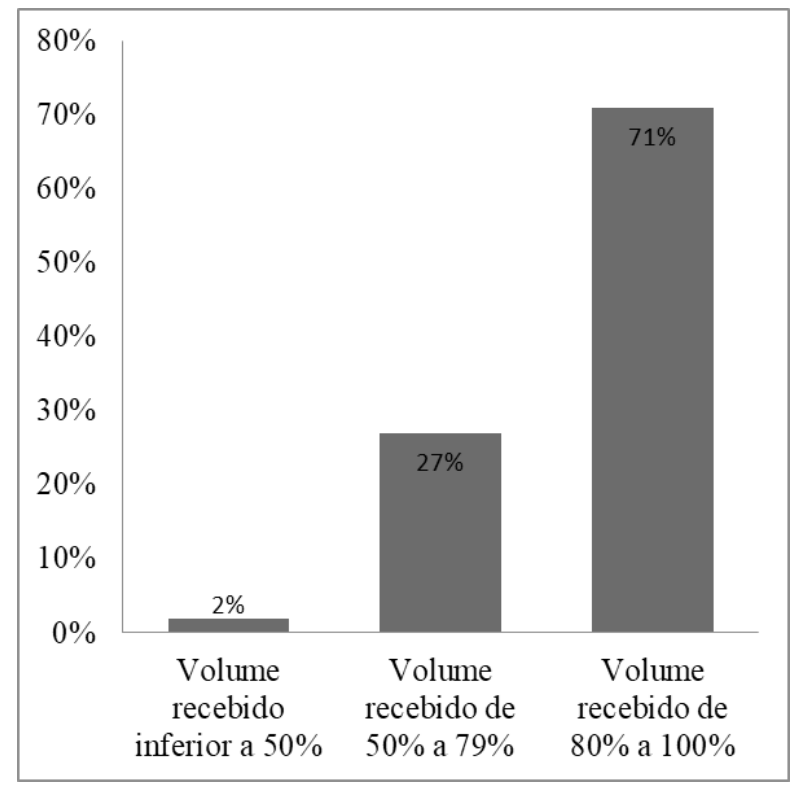

Fonte: Elaboração própria.

Ao longo do período de acompanhamento foram registradas 222 interrupções de infusão de NE, das quais 14\% decorreram por complicações com a sonda nasoentérica (saída e obstrução), 12\% por instabilidade hemodinâmica do paciente e $12 \%$ por estase gástrica. Em $16 \%$ das interrupções a justificativa não estava registrada (Tabela 1 ).

Tabela 1 - Distribuição das causas de interrupções na Infusão da Nutrição Enteral em Unidades de Terapia Intensiva. Belo Horizonte, Minas Gerais, Brasil - 2017. (N=79)

\begin{tabular}{lc}
\hline Variáveis & Percentual (\%) \\
\hline Sem motivo justificado & 16 \\
Complicação com a sonda nasoenteral & 14 \\
Instabilidade hemodinâmica & 12 \\
Volume residual gástrico & 12 \\
Exames & 11 \\
Traqueostomia/Cirurgia & 9 \\
Vazão em Bomba de Infusão Contínua < Vazão Prescrita & 9 \\
Complicação do Trato Gastrointestinal (vômito/diarreia) & 6 \\
Raio-X & 5 \\
Outros motivos & 4 \\
Extubação & 2
\end{tabular}

Fonte: Elaboração própria.

Acerca do desfecho dos pacientes acompanhados, 63,2\% receberam alta da UTI, 7,7\% restituíram a nutrição por via oral e 29,1\% faleceram.

\section{Discussão}

Os resultados encontrados mostram que o uso da terapia nutricional enteral nos pacientes em terapia intensiva na Instituição onde se deu a coleta de dados pode ser considerado, de maneira geral, satisfatório. A terapêutica foi iniciada precocemente na maioria dos casos acompanhados, mostrando ser valorizada, e essa conduta foi capaz de contribuir efetivamente para a recuperação do paciente. Além disso, a rapidez na iniciação dessa terapia contribui para 
a prevenção de consequências danosas provenientes do jejum prolongado, que pode ocasionar translocação bacteriana e de toxinas, aumento da permeabilidade da mucosa intestinal, hipercrescimento bacteriano, hipermetabolismo em resposta à lesão e liberação de mediadores pró-inflamatórios $^{(4-5,8)}$. Em estudo desenvolvido com 32 pacientes, somente 50\% tiveram início da nutrição enteral iniciada precocemente ${ }^{(9)}$. Já em outro estudo, contudo, encontrou-se percentual superior aos resultados desta pesquisa, no qual 77,2\% também tiveram início precoce da infusão ${ }^{(10)}$, dando indícios de que esse percentual, na instituição pesquisada, pode ser melhorado.

Além de ter início precoce, os resultados mostraram que $71 \%$ dos pacientes receberam 80 a 100\% do aporte planejado, um resultado superior à maioria dos resultados de estudos semelhantes disponíveis na literatura. Em um deles, no qual foram analisados o volume total de nutrição enteral recebido por 55 participantes e em que foi considerado como adequado a administração de no mínimo 90\% do volume prescrito, observou-se que apenas $42 \%$ dos pacientes receberam o volume mínimo esperado da nutrição enteral, sinalizando que a maioria não alcançou 90\% do volume total almejado ${ }^{(11)}$. Em outro estudo, somente $36,1 \%$ dos pacientes alcançaram um valor $\geq 90 \%$ de adequação do volume prescrito $^{(6)}$, e em outro, apenas $48 \%$ chegou ao aporte planejado $^{(12)}$.

Mesmo sendo minoria, há estudos que mostram maior proximidade da meta planejada para a infusão. Em um deles, em que foram avaliados 53 pacientes adultos em unidade de terapia intensiva, encontrou-se que os pacientes receberam $82,7 \%$ do volume prescrito ${ }^{(13)}$. Em outro trabalho, em que foram avaliados 93 pacientes, encontrou-se uma adequação média de $82,6 \%$ do volume infundido da nutrição enteral $^{(14)}$. Cabe pontuar, entretanto, que nesses estudos não ficaram claros se o cálculo de infusão foi feito antevendo pausas para a realização de procedimentos diários, como banhos e curativos, bem como para fisioterapia, o que pode ocasionar diferenças na avaliação dos achados.
Em se tratando de pacientes internados em unidade de terapia intensiva, interrupções na infusão da nutrição enteral decorrentes de intercorrências clínicas e hemodinâmicas têm alta probabilidade de ocorrência diante da gravidade do quadro clínico dos pacientes, o que dificulta alcançar 100\% de efetividade no balanço final da infusão de nutrição enteral ${ }^{(15)}$. Neste estudo, a instabilidade clínica e hemodinâmica foi responsável por 12\% das interrupções; nesses casos, não há alternativa senão interromper a infusão da nutrição enteral, uma vez que sua continuidade ocasionaria maiores riscos aos pacientes, como de aspiração de conteúdo gástrico.

Contudo, um percentual significativo de interrupções na infusão da nutrição enteral ocorreu também por outros motivos, sendo os mais prevalentes problemas mecânicos com a sonda nasoenteral (14\%), seguido por intercorrências relacionadas à tolerância da nutrição enteral, com presença de volume residual gástrico (12\%), e ainda relacionados à realização de exame (11\%), achados que corroboram resultados de outros trabalhos publicados, variando em suas proporções ${ }^{(12-13)}$. Em um deles, a principal causa de interrupção foi o jejum para realização de procedimentos (20,2\%), diferindo dos achados deste estudo, havendo 18\% de intercorrências relacionadas à sonda nasoenteral e 15,9\% a resíduo gástrico, além de procedimentos da unidade de terapia intensiva (11,5\%) e instabilidade hemodinâmica $(9,3 \%)^{(13)}$. As complicações gastrointestinais, como resíduo gástrico elevado, diarreia, vômito e constipação, agrupadas, representaram 26,8\% das complicações ${ }^{(13)}$. Em outra pesquisa as causas de interrupção foram, em sua maioria, problemas mecânicos com o cateter (36\%), seguidos por instabilidade hemodinâmica ou clínica (33\%), complicações do trato gastrointestinal (15\%), jejum para exames (12\%) e jejum para procedimentos e cirurgias $(4 \%)^{(12)}$.

Mesmo apresentando resultado melhor se comparado a outros estudos no que tange às complicações mecânicas com a sonda nasoenteral, trata-se de uma intercorrência passível de prevenção com cuidados de enfermagem e que pode alcançar percentuais ainda menores 
com a capacitação da equipe. A exteriorização da sonda nasoenteral pode decorrer de agitação psicomotora, distúrbio neurológico ou durante a locomoção do paciente, como ainda por tosse, náuseas ou vômitos, ou retirada ${ }^{(3,16)}$, situações essas que podem ser discutidas e controladas com atuação multiprofissional, visando evitar danos ao paciente. Um cuidado essencial para a prevenção da perda da sonda nasoenteral está na avaliação diária da fixação do cateter nasoenteral com os cuidados ao manipular o paciente, bem como protegendo seu posicionamento externo de forma a dificultar sua retirada pelo paciente $^{(3)}$.

Dentre as intercorrências mecânicas relacionadas ao uso da sonda nasoenteral, destaca-se a sua obstrução, que decorre quase sempre da falta de irrigação com água em momentos necessários e/ou de forma periódica, como antes de iniciar e ao término da infusão da dieta, e antes e após a infusão de medicamentos. Pode decorrer, ainda, da interação entre medicamentos ou entre medicamentos e a nutrição enteral, o que deve ser discutido com a equipe multiprofissional, mediante consulta à literatura disponível sobre o assunto, a fim de evitar interações favorecedoras das obstruções ${ }^{(3,16-17)}$.

Quanto às interrupções relacionadas à estase gástrica, neste estudo, os resultados foram mais difíceis de serem comparados com a literatura, uma vez que pouco foi citada como causa de interrupção de infusão de forma isolada, estando incluída, muitas vezes, na categoria de complicações gastrointestinais de forma ampliada. Em um estudo no qual o volume residual gástrico foi descrito de forma isolada, encontrou-se percentual um pouco superior (15,9\%) quando comparado aos achados desta pesquisa $(12 \%)^{(13)}$.

Cabe pontuar que na instituição onde a pesquisa foi realizada, o protocolo institucional vigente no momento da coleta de dados preconizava, para pacientes internados na UTI, a verificação de estase gástrica a cada 6 horas e orientava a interrupção, caso o volume encontrado fosse igual ou maior a $250 \mathrm{ml}$. Era preconizado ainda que fossem aguardadas 3 horas até que nova avaliação do paciente fosse realizada, para só então reiniciar a nutrição enteral.

Destaca-se que esse protocolo estava sendo revisado ao final da coleta dos dados pela Equipe Multiprofissional de Terapia Nutricional (EMTN) da instituição, com propensão à sua suspensão, atendendo às recomendações da American Societyof Parenteral e Enteral Nutrition (ASPEN) e a European Society of Parenteral e Enteral Nutrition (ESPEN), que recomendam que o monitoramento do volume residual gástrico não seja rotina em pacientes críticos. Esta conduta está indicada em situações específicas, e a suspensão da NE só deve ocorrer em volumes superiores a $500 \mathrm{ml}$, quando outras estratégias tiverem sido adotadas sem resultados positivos ${ }^{(18-19)}$.

Em estudo realizado no Japão, encontrou-se 14\% de intercorrências gastrintestinais e 13\% de diarreia. Essas intercorrências também se configuram como problemas relacionados à tolerância da dieta, assim como a presença de volume residual gástrico, devendo ser discutidos em equipe e avaliado o uso de pró-cinéticos, bem como a fórmula utilizada e a velocidade de infusão, a fim de contribuir para que sejam sanadas ${ }^{(20)}$.

Poucos estudos citaram problemas relacionados à falta de registros sobre as causas de interrupção, como encontrado nesta pesquisa (16\%). Esse resultado sinaliza a existência de problemas de comunicação efetiva entre a equipe, o que acaba por comprometer não só a segurança e qualidade assistencial, mas também a gestão do cuidado, salientando-se ainda que registros completos e claros sobre a situação do paciente e intercorrências são obrigação profissional previstos em legislação. Estudo realizado com pacientes internados em unidade de terapia intensiva e enfermarias concluiu que interrupções frequentes na infusão da nutrição enteral sinalizam falta de consciência acerca da importância da terapia nutricional enteral pelos profissionais de saúde e mostram falhas de comunicação na equipe $^{(21)}$, o que se corrobora neste estudo.

Quanto à interrupção decorrente de jejum para a realização de exames e procedimentos, trata-se de causa difícil de ser evitada, devendo, contudo, ser considerada tanto ao se planejar a 
infusão da terapia nutricional enteral quanto ao se avaliar o balanço da infusão nessas unidades. Entretanto, essas interrupções devem ocorrer o mais brevemente possível, com solicitação de agilização dos procedimentos/exames e determinação de jejum no menor período de tempo necessário. Cabe citar que na atualidade formas de confirmação do posicionamento mais rápidas têm sido testadas, como por meio de verificação do $\mathrm{pH}$, feito pela enfermagem à beira do leito, o que pode contribuir para a celeridade da infusão da nutrição enteral, uma vez que dispensa a realização de radiografia e o tempo perdido com sua realização ${ }^{(22)}$.

Soma-se aos fatores relacionados à efetividade da infusão da nutrição enteral falhas na programação da bomba de infusão e atrasos ao se iniciar a infusão da nutrição enteral. São resultados que reforçam a necessidade de educação permanente da equipe de enfermagem, com base em recomendações atuais e em evidências voltadas para a prática da terapia nutricional segura e com qualidade, uma vez que é uma terapêutica que envolve riscos e que requer equipe capacitada para sua realização ${ }^{(23)}$.

Acredita-se que os resultados desta pesquisa possam colaborar para melhorias no planejamento da assistência, não só para a unidade de terapia intensiva da Instituição na qual os dados foram coletados, mas também para outras instituições, inclusive por permitir a comparação dos achados. Neste estudo privilegiou-se comparar os achados com a literatura nacional tendo em vista a maior similaridade com as condições de trabalho das unidades de terapias intensivas. Nos países norte-americanos e europeus, por exemplo, a composição da equipe de enfermagem difere muito do contexto brasileiro, no qual há uma divisão técnica dentro da enfermagem com a atuação de técnicos de enfermagem, e não somente de profissionais graduados, como ocorre em outros países.

\section{Conclusão}

Os achados deste estudo permitem afirmar que a infusão da NE nos pacientes em UTI pode ser considerada satisfatória, com início precoce dessa terapêutica e aporte alcançado na infusão da nutrição enteral próximo do planejado. Esses dados sinalizam a qualidade da equipe assistencial, composta majoritariamente por enfermeiros graduados, bem como pela atuação da Equipe multiprofissional de terapia nutricional.

Contudo, os achados mostram também pontos frágeis a serem melhorados, em específico no que diz respeito à prevenção de complicações relacionadas ao uso da sonda nasoenteral, bem como nos registros relacionados às causas de interrupção da dieta. As informações desta pesquisa poderão subsidiar capacitações da equipe de enfermagem, visando o aprimoramento da prática assistencial não só na instituição onde foi realizado o estudo, mas em outras instituições. Sugere-se a realização de novos estudos acerca desta temática diante da importância de ser assegurada a efetividade da infusão da nutrição enteral nos diferentes serviços, visando a manutenção do estado nutricional dos pacientes em terapia intensiva, condição essencial à sua recuperação na totalidade.

\section{Contribuições:}

1 - concepção, projeto, análise e interpretação dos dados: Sterline Therrier, Clara Marize Carlos, Raquel Fernandes Costa e Jaqueline Almeida Guimarães Barbosa;

2 - redação do artigo e revisão crítica relevante do conteúdo intelectual: Sterline Therrier, Clara Marize Carlos, Raquel Fernandes Costa, Giovana Paula Rezende Simino e Jaqueline Almeida Guimarães Barbosa;

3 - aprovação final da versão a ser publicada: Sterline Therrier, Clara Marize Carlos, Raquel Fernandes Costa, Giovana Paula Rezende Simino e Jaqueline Almeida Guimarães Barbosa.

\section{Referências}

1. Correia MITD, Perman MI, Waitzberg DL. Hospital Malnutrition in Latin America: A systematic review. Clin Nutr. 2017;36(4):958-67. DOI:10.1016/j.clnu.2016.06.025 
2. Vallejo KP, Martínez CM, Adames AA, Fuchs-Tarlovsky V, Nogales GCC, Paz RER, et al. Current clinical nutrition practices in critically ill patients in Latin America: a multinational observational study. Crit Care. 2017;34(4):969-75. DOI:10.1186/s13054-017-1805-Z

3. Matsuba C, Magnoni D. Enfermagem em Terapia Nutricional. São Paulo: Sarvier; 2009.

4. Cirilo SMA, Nascimento CX, Sousa BS, Silva PFOA, Silva JE, Bandeira GFS. Impacto da terapia nutricional enteral precoce sob o tempo em uso de ventilação mecânica invasiva de pacientes críticos. Nutr clín diet hosp. 2018;38(3):149-53. DOI: $10.12873 / 383$ marry

5. Singer $\mathrm{P}$, Blasen A, Berger $\mathrm{M}$, Alhazzani W, Calder P, Casaer M, et al. ESPEN guideline on clinical nutrition in the intensive care unit. Clin Nutr. 2018;37(3):1-32. DOI:10.1016/j.clnu.2018.08.037

6. Stefanello MD, Poll FA. Estado nutricional e dieta enteral prescrita e recebida por pacientes de uma Unidade de Terapia Intensiva. ABCS Health Sci. 2014;39(2):71-6. DOI:10.7322/abcshs.v39i2.625

7. Couto CFL, Moreira JS, Hoher JA. Terapia nutricional enteral em politraumatizados sob ventilação mecânica e oferta energética enteral. Rev Nutr. 2012;25(6):695-705. DOI: 10.1590/ S1415-52732012000600002

8. Bezerra GKDA, Cabral PC. Nutrição enteral precoce em pacientes críticos e sua associação com variáveis demográficas, antropométricas e clínicas. Braspen J [Internet]. 2018 [cited 2020 Jun 6];33(4):446-50. Available from: http://arquivos. braspen.org/journal/out-dez-2018/artigos/14-AONutricao-enteral-precoce.pdf

9. Gonçalves $\mathrm{CV}$, Borges LR, Orlandi SP, Bertacco RTA. Monitoramento da Terapia Nutricional Enteral em Unidade de Terapia Intensiva: Adequação calórico-proteica e sobrevida. Braspen J [Internet]. 2017 [cited 2020 Jun 10];32(4):341-6. Available from: http:// arquivos.braspen.org/journal/out-dez-2017/08Monitoramento-da-terapia.pdf

10. Medeiros IMS, Ritter CG, Ribeiro Filho GHC, Prado PR. Caracterização e adequação energético-proteica da nutrição enteral em pacientes em uma unidade de terapia intensive: Nutrição enteral no paciente crítico. South Am J Basic Educ, Tech Technol [Internet]. 2020 [cited 2020 Jun 10];6(2):381-95. Available from: https:// periodicos.ufac.br/index.php/SAJEBTT/article/ view/2910/2063
11. Silva GL. Adequação do volume de nutrição enteral administrado em relação ao prescrito e causas para a inadequação na unidade de terapia intensiva de um hospital universitário no triângulo mineiro [dissertação na internet]. Uberaba (MG): Universidade de Uberaba; 2014 [cited 2020 Jun 12]. Available from: https://www.uniube.br/propepe/ ppg/odontologia/arquivos/2014/dissertacoes/ Gabriela.pdf

12. Mendonça MR, Guedes G. Terapia nutricional enteral em uma Unidade de Terapia Intensiva: prescrição versus infusão. Braspen J [Internet]. 2018 [cited 2020 Jun];33(1):54-7. Available from: http://arquivos.braspen.org/journal/jan-fev-mar2018/10-AO-Terapia-nutricional-enteral.pdf

13. Rocha A, Oliveira A, Cabral N, Gomes R, Guimarães T, Wanderson B, et al. Causas de interrupção de nutrição enteral em unidades de terapia intensiva. Rev Pesq Saúde [Internet] 2017 [cited 2020 Jul 29]; 18(1):49-53. Available from: http://www.periodicoseletronicos.ufma.br/index. $\mathrm{php} / \mathrm{revistahuufma/article/view/7880}$

14. Ribeiro LMK, Oliveira Filho RS, Caruso L, Lima PA, Damasceno NRT, Soriano FG. Adequação dos balanços energético e proteico na nutrição por via enteral em terapia intensiva: quais os fatores limitantes? Rev bras ter intensiva. 2014;26(2):155-62. DOI:10.5935/0103-507X.20140023

15. Gambato J, BoscainI C. Adequação da prescrição dietética e sua associação com intercorrências em pacientes em uso de terapia nutricional enteral. Rev Bras Nutr Clin. [Internet]. 2017 [cited 2020 Jul 29];30(4):338-43. Available from: http://www.braspen.com.br/home/wp-content/ uploads/2016/11/13-Adequa\%C3\%A7\%C3\%A3oda-prescri\%C3\%A7\%C3\%A3o.pdf

16. Freitas TECD, Ferreira $\mathrm{CH}$, Bezerra JL, Oliveira KAB, Carvalho LRD, Sales ALDCC. Avaliação dos indicadores de qualidade da terapia de nutrição enteral em um hospital universitário. Braspen J [Internet]. 2018; [cited 2020 Jun];33(4):395-401. Available from: http://arquivos. braspen.org/journal/out-dez-2018/artigos/07-AOAvaliacao-dos-indicadores.pdf

17. Ferre R, Joaquim FM, Pavan AM, Parra BFCS, Matos LBN, Silva Júnior AV, et al. Manual de administração de medicamentos por acessos enterais. Braspen J [Internet]. 2019 [cited 2020 Jul 29];34(2)193-212. Available from: http://arquivos. braspen.org/journal/abr-mai-jun-2019/artigos/14Manual-de-diuicao.pdf 
18. McClave S, Taylor B, Martindale R, Warren M, Johnson D, Braunschweig C, et al. Guidelines for the provision and assessment of nutrition support therapy in the adult critically Ill patient: Society of Critical Care Medicine (SCCM) and American Society for Parenteral and Enteral Nutrition (A.S.P.E.N). JPEN J Parenter Enteral Nutr. 2016;40(2):159-11. DOI: $10.1177 / 0148607115621863$

19. Singer P, Blaser AR, Berger MM, Alhazzani W, Calder PC, Casaer MP, et al. ESPEN guideline on clinical nutrition in the intensive care unit. Clin Nutr. 2019;38(1):48-79. DOI:10.1016/j.clnu. 2018.08.037

20. Uozumi M, Sanui M, Komuro T, Iisuka Y, Kamio T, Koyama $\mathrm{H}$, et al. Interruption of enteral nutrition in the intensive care unit: a single-center survey. J Intensive Care. 2017;5(52). DOI: 10.1186/ s40560-017-0245-9

21. Martins JR, Shiroma GM, Horie LM, Logullo L, Silva MLT, Waitzberg DL. Factors leading to discrepancies between prescription and intake of enteral nutrition therapy in hospitalized patients. Nutrition. 2011;28(9):864-7. DOI: 10.1016/j.nut. 2011.07 .025

22. Ester MPF, Siok BT, Shin YA. Nasogastric tube placement confirmation: where we are and where we should be heading. Proc Singapore Healthcare. 2017;26(3):189-95. DOI: $10.1177 / 2010105817705141$

23. DiMaria-Ghalili RA, Gilbert K, Lord L, Neal T, Richardson D, Tyler R, et al. Standards of Nutrition Care Practice and Professional Performance for Nutrition Support and Generalist Nurses. Nutri Clin Pract. 2016;31(4):527-47. DOI: $10.1177 / 0884533616653835$

Recebido: 25 de agosto de 2020 Aprovado: 29 de setembro de 2020

Publicado: 23 de novembro de 2020

A Revista Baiana de Enfermagem utiliza a Licença Creative Commons - Atribuição-NãoComercial 4.0 Internacional. https://creativecommons.org/licenses/by-nc/4.0/ Este artigo é de acesso aberto distribuído sob os termos da Licença Creative Commons (CC BY-NC). Esta licença permite que outros remixem, adaptem e criem a partir do seu trabalho para fins não comerciais. Embora os novos trabalhos tenham de lhe atribuir o devido crédito e não possam ser usados para fins comerciais, os usuários não têm de licenciar esses trabalhos derivados sob os mesmos termos. 